\title{
Anglizismen im öffentlich-medialen und sprachwissenschaftlichen Diskurs über die deutsche Sprache und in den Einstellungen der Sprecher
}

\author{
Goranka Rocco (Bologna)
}

\begin{abstract}
This article explores the discourse on anglicisms in German. The first part is dedicated to the assumptions of language specialists and non specialists regarding foreign words and especially anglicisms, language change and language deterioration. These aspects are analysed with regard to different moments in the recent history of German. The second, empirical part of the article presents the results of an extensive questionnaire study among German university students regarding their attitudes towards the current language situation and the use of anglicisms in German. The results of this study are compared to the results of other, similar studies on attitudes towards language change, language deterioration and anglicisms in German.
\end{abstract}

\section{$1 \quad$ Einleitung}

Das Verhältnis zwischen Spracheinstellungen und dem öffentlichen Diskurs über Sprache ist schon allein aufgrund der hypothetischen Natur des Konstrukts Einstellung (attitude) ${ }^{1}$ und der Mannigfaltigkeit des öffentlichen Sprachdiskurses kein einfaches.

Der öffentlich-mediale epilinguistische ${ }^{2}$ Diskurs hat vielfältige Erscheinungsformen und Themenschwerpunkte und kann in Abhängigkeit der gesellschaftspolitischen Entwicklungen stark variieren. Er verschmilzt gelegentlich mit publizistischer Sprachkritik und nährt oft Topoi, u. a. den Sprachverfallstopos und die damit verwandten Topoi vom bedrohlichen Fremdspracheneinfluss und von der sinkenden Sprachkompetenz Jugendlicher (vgl. Spitzmüller 2005: 2, 8f.; Sitta 1990). Diese schlagen sich wiederum als kollektives "Wissen" in den individuellen Spracheinstellungen nieder. ${ }^{3}$

Eine begrenzte Zahl von Sprechern, zu der u. a. Studierende philologischer Fachrichtungen gehören, kommt allerdings nicht nur in Berührung mit dem öffentlich-medialen, sondern auch

\footnotetext{
${ }^{1}$ Die Eistellung geht schon aus einer der ersten und in der Literatur weitgehend akzeptierten Definitionen von Allport als hypothetisches Konstrukt hervor: Es handelt sich um eine mentale Größe, die sich der unmittelbaren Beobachtung entzieht: "An attitude is a mental and neural state of readiness, organized through experience, exerting a directive or dynamic influence upon the individual's response to all objects and situations to which it is related" (Allport 1935: 810, vgl. auch Upshaw 1975: 203). Für einen Überblick über die Definitionen vgl. Rocco (2010: 15-17).

${ }^{2}$ Die Begriffe epilinguistisch und besonders metalinguistisch sind in ihrer Verwendung teilweise mehrdeutig. Hier wird konsequent der Begriff epilinguistisch als Bezeichnung für den subjektiven, nichtlinguistischen Diskurs über Sprachen, Sprachgebrauch und Sprecher im Sinne der besonders in der französischen Soziolinguistik untersuchten représentations und des imaginaire linguistique (vgl. z. B. Canut 1993, 1998; Bavoux 1997) verwendet.

3 Das komplexe Verhältnis zwischen Sprachentwicklung, Spracheinstellungen und dem öffentlichen Diskurs thematisiert auch Eisenberg (2011: 9-15).
} 
mit dem linguistischen Sprachdiskurs. Im Laufe ihres Studiums setzen sich SprachenStudierende in der Regel mehr oder weniger tiefgehend mit der linguistischen Sichtweise auseinander; und es kann angenommen werden, dass sich die Dialektik der Sprachdiskurse auf die (v. a. kognitive ${ }^{4}$ Komponente der) Spracheinstellungen auswirkt.

Vor diesem Hintergrund können nun die Ziele der vorliegenden Untersuchung formuliert werden: Es geht einerseits um das Spannungsverhältnis zwischen dem epilinguistischen und dem linguistischen Diskurs, das mit Blick auf einige Momente der deutschen Sprachgeschichte sowie auf andere Sprachen beleuchtet (1) und anschließend speziell im Hinblick auf die Anglizismen im Deutschen untersucht wird (2). Andererseits wird ein Versuch unternommen, sich der Frage nach dem Einfluss beider Diskurse auf die Spracheinstellungen auf empirischem Wege zu nähern: Hierzu werden die empirisch erhobenen Spracheinstellungen der Studierenden mit den in anderen Studien ermittelten Spracheinstellungen der Gesamtbevölkerung verglichen (3).

\section{Sprache zwischen öffentlicher und sprachwissenschaftlicher Perspektive und Ideologisierung der Sprachpflege im nationalen und historischen Vergleich}

Fremdwörtergebrauch, "korrekter" Sprachgebrauch, Abgrenzung von Varietäten einer Sprache oder von Standard und Dialekt, Genus und Sexus, Rechtschreibregeln und -kompetenzen usw. gliedern sich in eine lange Reihe der sprachbezogenen Themen ein, die aufgrund eines generellen Gefühls des Beteiligt- oder Betroffenseins gern und häufig in der Öffentlichkeit diskutiert werden. Wie ist es aber um die Bereitschaft der eigentlichen Sprachexperten bestellt, an diesen Diskussionen teilzunehmen?

Die Frage des Dialogs zwischen dem öffentlichen Sprachdiskurs und der linguistischen Perspektive ${ }^{5}$ wurde u. a. auch im Rahmen der vom Goethe-Institut Moskau und dem PuschkinInstitut Moskau veranstalteten Moskauer Konferenz Die Rolle der europäischen Sprachen in der Zukunft - deutsch-russische Erfahrungen und Perspektiven 2007 erörtert, zu deren zentralen Anliegen die nähere Erkundung des Verhältnisses zwischen Sprachverfall und Sprachentwicklung zählte. Dieses Verhältnis war auch Gegenstand der 2013 abgehaltenen IDSTagung Sprachverfall? Dynamik - Wandel - Variation (vgl. Hein/Mell 2013).

In seinem Bericht, der das Fazit der Auseinandersetzung mit diesem Thema in Moskau liefert, betont Eisenberg, dass sich die Sprachwissenschaft am öffentlichen Diskurs über Sprache zu beteiligen hat: "Ihr Beitrag besteht darin,", so Eisenberg (Ammon/Eisenberg/Scholz 2008: 67), "den Diskurs zu versachlichen und über Sinn wie Erfolgsaussichten sprachpflegerischer Maßnahmen Auskunft zu geben". ${ }^{6}$

Es scheint jedoch, dass ein solcher Austausch in der deutschen Öffentlichkeit in einem eher beschränkten Umfang stattfindet (zumindest gemessen an den gegebenen technischen Möglichkeiten und an den Dimensionen anderer Debatten). Aus dieser Sicht können beispielsweise die sprachpflegerischen Versuche von Bastian Sick geradezu als ein Glücksfall bezeichnet werden, da sie mehrere Wissenschaftler zu öffentlicher Kritik bewogen haben und den Anlass lieferten, das Verhältnis zwischen populärer und sprachwissenschaftlicher Sichtweise auch mit Blick auf die Unterhaltungsfunktion der populären Sprachkritik in den

\footnotetext{
${ }^{4}$ Die hypothetische Natur der Einstellung ist auch einer der Gründe für die Tatsache, dass die Meinungen hinsichtlich der Einstellungsstruktur z. T. auseinandergehen (vgl. Rocco 2010: 15-17). In Anlehnung an Katz und Stotland (1959) wird hier von der Annahme ausgegangen, dass der Anteil an affektiven, kognitiven und konativen Elementen je nach Einstellungsobjekt variieren kann.

5 Vgl. Spitzmüller 2005 und auch weiter unten.

${ }^{6}$ Zur linguistischen Stellungnahme zu den Thesen zur "Bedrohung" bzw. zum "Verfall" des Deutschen durch Anglizismen vgl. auch Eisenberg 1999, Schlobinski 2000.
} 
Mittelpunkt zu rücken. ${ }^{7}$ Dabei wurde besonders auch die Tendenz zur Verschmelzung bzw. Verwechslung der Sphären des Fachdiskurses einerseits und des Laien- bzw. "Entertainerdiskurses" andererseits thematisiert. Ágel bringt diese Problematik auf den Punkt mit der Frage: "Was tun, wenn für das Publikum der Entertainer auch als eine Art normative Instanz, die verständlicher ist als der Duden, in Frage kommt?" (Ágel 2008: 81).

Es kann angenommen werden, dass divergierende Grundeinstellungen im Hinblick auf die Dichotomien präskriptiv vs. deskriptiv und subjektiv-wertend vs. objektiv-neutral (exemplarisch ausgedrückt in der Gegenüberstellung von Sprachverfall und Sprachentwicklung) das Zustandekommen eines quantitativ und qualitativ ausgewogenen Dialogs teilweise verhindern. Mit der Divergenz der Sichtweisen hängt als ein weiterer Faktor die Tatsache zusammen, dass die Schnittmenge der potenziellen Diskussionsgegenstände eher begrenzt ist: Die Schwerpunkte der öffentlichen Sprachdiskussion liegen, wie eingangs angedeutet, größtenteils im Bereich der Sprachpflege und Sprachnorm, die nur zwei der möglichen Untersuchungsobjekte der Sprachwissenschaft bilden. Und schließlich kann die Diskussionsbereitschaft der Sprachwissenschaftler und ihr Selbstverständnis als Linguisten und zugleich Teil einer Gesellschaft in Abhängigkeit von historischen und geopolitischen Grundbedingungen, von der aktuellen gesellschaftspolitischen Stimmung und nicht zuletzt vom Grundton der Diskussion variieren. ${ }^{8}$

In diesem Zusammenhang muss das Problem der Ideologisierung der Sprachpflege angeschnitten werden. Dabei ist nicht nur an sprachpolitische Extremsituationen wie etwa die der Hitler- oder der Mussolini-Ära ${ }^{9}$ zu denken, wo eine Konvergenz mit der öffentlichen oder politischen Meinung der Stunde oder eine Abweichung von derselben klare Vor-. bzw. Nachteile für die Karriere oder gar für die persönliche Unversehrtheit implizierte, sondern auch an Momente der Selbstdefinition und Versuche der Abgrenzung von Völkern bzw. von sprachlichen, territorialen oder kulturellen Gemeinschaften, oder an Situationen, in denen von einer mehr oder weniger konstanten Wahrnehmung des "Bedrohtseins" gesprochen werden kann.

Dass die Perzeption der sprachpolitischen Lage eine mehr oder minder starke Ideologisierung der Sprachpflege - und oft auch der Sprachwissenschaft - zu legitimieren scheint, kann an zahlreichen Beispielen aus anderen gesellschaftlichen Kontexten illustriert werden. So stellt

\footnotetext{
7 So kritisiert z. B. Ágel (2008) eine Reihe von methodischen und anderen Problemen wie z. B. die Verwechslung von Problemen der Kasuskategorie mit denen der Kasusform (vgl. ebd. 74), die irreführende Darstellung des Dativobjekts als typische Konkurrenzform des Genitivobjekts (vgl. ebd. 77), sowie allgemeiner die fehlende Sensibilität für Sprachwandelprozesse und für Teilsysteme, die marginale Rolle und Unsystematizität der Sprachgebrauchsanalysen (vgl. ebd. 81). Eisenberg problematisiert u. a. Sicks Behauptungen bzgl. des "richtigen" und "falschen" Partizips von winken und der "richtigen" und "falschen" Deklination des Demonstrativums im Adverbial am Anfang dieses/diesen Jahres (vgl. SpiegelKritik 2006). In einem Interview mit dem Goethe-Institut interpretiert er das Phänomen als symptomatisch für die aktuelle Ratgeberkultur bzw. "den allgemeinen Ratgeberboom" und die Suche nach Entertainment (vgl. Giersberg 2007). Zur Kritik an populärwissenschaftlichen Ausführungen vgl. auch Maitz/Elspaß 2007; Schneider 2005, 2008; Schümann 2007; Topalović/Elspaß 2008.

8 Schneider (2006) sieht einen Zusammenhang zwischen der geringen Beteiligung der Linguisten am normativen Diskurs und dem Erfolg der populärwissenschaftlichen Literatur: "An der Tatsache, dass Publikationen dieser Qualität derzeit den öffentlichen Diskurs über Sprache beherrschen, ist die Linguistik alles andere als unschuldig. In ihrem Selbstverständnis, eine ausschließlich deskriptive Wissenschaft zu sein, hat sie das Normativitätsproblem jahrzehntelang trivialisiert und muss sich nun nicht wundern, wenn sie zum Thema Sprachrichtigkeit kaum noch befragt wird." Spitzmüller (2005: 15) nennt vielfältige Ursachen für die fehlende Kommunikation bzw. Verständigung zwischen Sprachwissenschaftlern und der Öffentlichkeit, u. a. den allgemeinem Legitimationsverlust der Wissenschaften infolge zunehmender Thesenpluralität und die (aus zunehmender Spezialisierung resultierende) Unmöglichkeit der Vermittlung von Wissenschaftsinformationen.

9 Zur sprachpflegerischen Tätigkeit in der Zeit des Nationalsozialismus vgl. bes. Hillen (1982: 56-62), Polenz (1967); Riegel (2007: 8-13).
} 
Pavić Pintarić (2003) nach einer Auswertung der in den vergangenen fünf Jahren erschienenen Publikationen der bedeutendsten kroatischen Sprachwissenschaftler fest, dass die meisten den (sich v. a. in der Haltung gegenüber Fremdwörtern manifestierenden) Purismus positiv, als traditionsbewahrend und z. T. als Synonym für Sprachkultur empfinden, was sich v. a. angesichts der geopolitischen Lage, der zahlreichen direkten oder indirekten Kontakte zu verschiedenen Sprachen, der geringen Verbreitung des Kroatischen und seiner vorwiegenden Rolle als Aufnahmesprache erklärt (vgl. auch Samardžija 1993: 7). Im Zusammenhang mit den Nachfolgerstaaten des ehemaligen Jugoslawien ist auch der Abgrenzungseifer bei der Konstituierung von neuen nationalen Standards zu erwähnen (vgl. Neweklowski 2010: 188206; Völkl 2002: 217; Gröschel 2009). Besonders ideologieanfällig sind der Sprachdiskurs und die Sprachpflegemaßnahmen im aktuellen Bosnien, wo sich oft ein- und dieselbe Sprache sprechende Bürger in verschiedenen Alltagssituationen gezwungen sehen, sich in ihrem Sprachgebrauch je nach ethnischer und/oder religiöser Zugehörigkeit an verschiedene Varietäten bzw. Standards anzupassen.

Ein weiteres Beispiel für ein stets latent vorhandenes Gefühl des "Bedrohtseins" liefert Québec: Sowohl im Hinblick auf die bedrohliche Anglophonie als auch auf das Verhältnis zwischen der eigenen Varietät und dem Französischen der Metropole scheint ständig die Notwendigkeit zu bestehen, sich anderen Sprachen gegenüber zu behaupten (vgl. z. B. Sarcher 1994; Wolf 1987; Gendron 1974).

Es lassen sich noch zahlreiche weitere Sprachkontexte nennen, die durch einen (kultur-)politisch und z. T. ethnisch begründeten, mehr oder weniger ideologiedurchsetzten Sprachdiskurs geprägt sind, die Sprachverhalten, Spracheinstellungen und z. T. auch linguistische Ansichten und Tätigkeit beeinflussen: etwa die auf die kulturpolitische Identitätsstärkung bedachten Bemühungen Norwegens seit der Auflösung der Union mit Dänemark 1814, das Nynorsk zu normieren (vgl. Pettersen 1989) oder die Südtiroler Diskussionen um Straßenschilder, Toponyme usw., die das seit dem Anschluss an Italien latent vorhandene Konfliktpotenzial von Zeit zu Zeit ansteigen lassen.

Wenn man sich nun aus dieser Perspektive an den aktuellen öffentlichen und institutionellen Diskurs über Fremdwörter im Deutschen annähert, so scheint er sowohl im Vergleich zu mehreren anderen Phasen der jüngeren deutschen Sprachgeschichte als auch im Vergleich zur aktuellen Sprachdiskussion in mehreren europäischen Ländern eher kultursoziologisch (vgl. Schlobinski 2000: 7) als politisch-ethnisch begründet zu sein: Seit der Gründung des Deutschen Kaiserreichs 1871 und auch noch in der Weimarer Zeit dient der puristische Sprachdiskurs, der die Aufgabe der Sprachpflege v. a. in der Bekämpfung von Fremdwörtern sieht, in erster Linie der Stärkung der politischen Einheit (vgl. Hillen 1982: 23-24; Riegel 2007: 7). In der NS-Zeit kann von einer extremen Ideologisierung des epilinguistischen sowie linguistischen Diskurses gesprochen werden, die bereits an Wendungen wie "arische Sprachgrundwerte", "Kriterium der Sprachechtheit", "Verehrung der Muttersprache als eines Volksheiligtums" (Hillen 1982: 61, 62; Riegel 2007: 8) ablesbar ist. In der Nachkriegszeit ist die Sprachpflege zwar nicht mehr antisemitisch oder allgemeiner rassistisch gefärbt, doch nach wie vor extralinguistisch begründet: Die Sorge um die deutsche Sprache und der allgegenwärtige Sprachverfall-Gedanke hängen stark mit den Kriegsfolgen wie Umsiedlung und Besatzung sowie mit der Sorge um den Untergang des Volkes zusammen (vgl. Riegel 2007: 13-14).

Wie stark sich der öffentliche und institutionelle Diskurs um Sprache und Sprachpflege inzwischen verändert hat, kann an dem von Riegel (vgl. ebd.: 14) angestellten Vergleich der Satzungen der Gesellschaft für deutsche Sprache (im Folgenden GfdS) illustriert werden: In der Satzung von 1947, als die GfdS in der Nachfolge des Allgemeinen Deutschen Sprachvereins gegründet wurde, ist die Rede von der Aufgabe, "dem deutschen Volk zu dienen". Die Arbeit an der deutschen Sprache wird dabei mit "Arbeit am deutschen Volkstum und an der deutschen Zukunft" gleichgesetzt (Hillen 1982: 87f.; Riegel 2007: 14), während in der aktuellen Satzung 
als erstes Ziel (Punkt 1) "Pflege und Erforschung der deutschen Gegenwartssprache"10 genannt wird.

Was andere europäische Länder und Sprachen betrifft, so bietet sich speziell im Hinblick auf die Anglizismen der Vergleich mit Frankreich bzw. mit dem Französischen als einer weiteren großen Sprache an, die in bestimmten Bereichen der internationalen Kommunikation vom Englischen verdrängt wurde: Mit Blick auf die französische Sprachpolitik kann mit Braselmann (2002: 306) angenommen werden, dass gesetzgeberische Eingriffe im Sinne des als Loi Toubon bekannten Gesetzes von 1994'11, das den Fremdwörter- und besonders Anglizismengebrauch eindämmen sollte, im aktuellen Deutschland sowie in mehreren anderen Ländern in dieser Form kaum durchsetzungsfähig wären. ${ }^{12}$ In der deutschen Politik hat es zwar einige Vorschläge im Sinne einer deutschen Variante der Loi Toubon gegeben, die jedoch ohne gesetzliche Konsequenzen blieben (vgl. Riegel 2007: 20). Vielmehr befürworten mehrere Sprachexperten im Dialog mit der Öffentlichkeit eine aktive, kompetente Zweisprachigkeit - als Antwort auf die Stellungsverluste des Deutschen in der Wissenschaftskommunikation und anderen Bereichen der internationalen Kommunikation (vgl. Ammon 2007), aber auch als Antwort auf die Wahrnehmung der "Bedrohung" des Deutschen durch Anglizismen bzw. des Englischen durch internationalen Nichtmuttersprachler-Gebrauch (vgl. Stein 2004).

Die Parallelen in den internationalen "Stellungsverlusten" der beiden Sprachen und in der wahrgenommenen Stärke des Englischeinflusses können sicher nicht verneint werden; ausschlaggebend für den Gestaltungsspielraum der Sprachpflegemaßnahmen scheinen jedoch eher die grundverschiedenen gesellschaftlich-historischen und kulturellen Bedingungen der beiden Länder zu sein.

\section{$3 \quad$ Anglizismen zwischen öffentlichem und linguistischem Diskurs}

Dass die aktuelle Sprachdiskussion in der deutschen Öffentlichkeit sowohl aus dem Vergleich zu anderen Ländern als auch aus dem Rückblick auf die eigene Vergangenheit generell als weniger politisiert bzw. ideologieverdächtig hervorgeht, heißt allerdings keineswegs, dass sie ganz undurchlässig für puristische Argumentation und Sprachverfall-Szenarien wäre und dass keine Gefahr der Verschmelzung oder Verwechslung von populärwissenschaftlicher und linguistischer Sicht besteht - ein selbstredendes Beispiel ist der bereits thematisierte Erfolg von Bastian Sick.

Von einem eher irreführenden als fruchtbaren Ineinandergreifen der beiden Sphären kann auch gesprochen werden, wenn Sprachwissenschaftler in den Medien nach ihrer Meinung gefragt werden und zwischen der Beantwortung der Frage und ihrer medialen Verarbeitung ein zweistufiger Prozess der Anpassung oder gar Verzerrung stattfindet: Der Experte wird von den Medien gefragt, wie er eine bestimmte sprachliche Entwicklung empfindet. Dabei sieht er sich teilweise aus der Rolle des objektiven Sprachbeobachters in die Rolle des fühlenden und

\footnotetext{
${ }^{10}$ Hier wird aus der aktuellen Satzung zitiert: www.gfds.de/wir-ueber-uns/satzung/.

$11 \mathrm{Zu}$ den Anglizismen im öffentlichen und medialen französischen Sprachgebrauch vgl. auch Plümer 2000. Im

Folgenden wird zur Veranschaulichung ein Artikel des genannten Gesetzes zitiert: "Toute inscription ou annonce apposée ou faite sur la voie publique, dans un lieu ouvert au public ou dans un moyen de transport en commun et destinée à l'information du public doit être formulée en langue française. [...] Si l'inscription rédigée en violation des dispositions qui précèdent est apposée par un tiers utilisateur sur un bien appartenant à une personne morale de droit public, celle-ci doit mettre l'utilisateur en demeure de faire cesser, à ses frais et dans le délai fixé par elle, l'irrégularité constatée. Si la mise en demeure n'est pas suivie d'effet, l'usage du bien peut, en tenant compte de la gravité du manquement, être retiré au contrevenant, quels que soient les stipulations du contrat ou les termes de l'autorisation qui lui avait été accordée“. Vgl. Loi n ${ }^{94-665 ~ d u ~} 4$ août 1994 relative à l'emploi de la langue française, Art. 3 (vgl. République Française 2014).

12 Im Zusammenhang mit der Plurizentrizität des Englischen zieht Stein (2004: 6) einen Vergleich zum Französischen und spricht von "seiner auch legislativ bewehrten, nicht mehr zeitgemäßen einheitlichen Korrektheitstyrannei."
} 
wertenden Sprachnutzers gedrängt, die er aber als konstruktiver Dialog- bzw. Interviewpartner und um sich den Sprecheransichten anzunähern teilweise akzeptiert. Für den zweiten Schritt sind die Medien zuständig, die seine Aussagen u. U. noch so umformulieren können, dass sie sich besser in den (oft gewollt dramatischen) medialen Diskurs fügen.

Um zwei konkrete, Anglizismen betreffende Beispiele anzuführen, die diesen Prozess andeutungsweise erkennen lassen: Der Germanistikprofessor und Vorsitzende des Deutschen Germanistenverbandes Jörg Kilian sagt vor der Presse anlässlich des Deutschen Germanistentages "Der Anglizismengebrauch muss die Menschen nicht besorgen, weil ihr Anteil am deutschen Wortschatz insgesamt relativ gering ist", betont ferner, dass sie nur rund $3 \%$ des Wortschatzes ausmachen und dass die deutsche Sprache auch schon früher von anderen Sprachen, etwa vom Lateinischen oder später vom Französischen, beeinflusst wurde. U. a. äußert er sich zum Anglizismengebrauch der deutschen Unternehmen, was anschließend von der dpa wie folgt wiedergegeben (und in dieser Form auch von mehreren Zeitungen und Zeitschriften übernommen) wird:

In der Werbesprache seien Anglizismen nicht immer sinnvoll, meinte Kilian. "Firmen wählen mitunter Anglizismen, um ihren eigenen Unternehmen einen internationalen Anstrich zu geben, bedenken aber nicht immer, dass sie damit bestimmt Kunden gar nicht mehr erreichen." Etwa weil man den Eindruck habe, "dass hier ein ziemlich rumpeliges Englisch gesprochen wird" (Fachverband Deutsch 2013).

Im entsprechenden Welt-Online-Artikel werden aber diese Stellungnahmen mit folgender Einleitung versehen:

Der Untergang der deutschen Sprache durch Anglizismen droht nicht - meint der Vorsitzende des Germanistenverbandes, Prof. Jörg Kilian. Er sorgt sich aber um den falschen Gebrauch von Fremdwörtern. ("Sprachexperte sieht in Anglizismen keine Gefahr") $)^{13}$

Die "Besorgnis" des eigentlich kaum als besorgt zu bezeichnenden Sprachwissenschaftlers, die im letzten oben zitierten Satz durchscheint, wird im letzten Textabschnitt noch einmal aufgegriffen:

Berechtigter sind die Sorgen um den Verfall der Sprache nach Ansicht Kilians eher, wenn man sich bestimmte werbestrategische Gebräuche von Anglizismen anschaut. "Firmen wählen mitunter Anglizismen, um ihren eigenen Unternehmen einen internationalen Anstrich zu geben, bedenken aber nicht immer, dass sie damit bestimmt Kunden gar nicht mehr erreichen." Etwa weil man den Eindruck habe, "dass hier ein ziemlich rumpeliges Englisch gesprochen wird". (ebd.)

Zweites Beispiel: In einem Spiegel-Online-Interview nach seiner Meinung über den Anglizismengebrauch gefragt, legt der emeritierte Germanist Karl-Heinz Göttert eine linguistisch begründete Sicht der Dinge dar: Er gibt ein entwarnendes, sachliches Urteil bezüglich der vom Journalisten ins Gespräch gebrachten "Sintflut von Anglizismen", weist auf den eher geringen Wortschatz-Anteil der Anglizismen hin, zitiert Scheinanglizismen wie Oldtimer und Handy als Beweise für kreativen Umgang der Nehmersprachen mit fremdem Lehngut, erwähnt Jacob Grimms ablehnende Haltung gegenüber dem Sprachpurismus. Er fügt allerdings auch eine persönlich gefärbte - wenn auch durch historische Umstände der Begriffsverwendung, und keineswegs etwa durch Anglizismen-Phobie begründete Anmerkung bezüglich des Lehnworts Blockbuster hinzu, wobei er eher die metaphorische Verwendung des Ausdrucks als den Anglizismus an sich problematisiert:

\footnotetext{
13 Vgl. "Sprachexperte sieht in Anglizismen keine Gefahr". Diese und die folgenden Kursiv-Hervorhebungen stammen von mir. Um Leseflussstörungen zu vermeiden, wurden zwei Fehler im Originaltext berichtigt: "meint der Vorsitzenden [sic] des Germanistenverbandes", "die Sorgen um den Verfall der Sprache da [sic] nach Ansicht".
} 
Ein Mountainbike ist wirklich kein klassisches Fahrrad der Art, wie ich es zum Beispiel fahre.

Das Wort ist also eine Bereicherung für die deutsche Sprache, die sich noch dazu faktisch durchgesetzt hat. Geschmacklos dagegen finde ich das Lehnwort Blockbuster, also Kinokassenschlager. Denn wir wissen, dass damit einmal Bomben bezeichnet wurden, die ganze Häuserblocks in Schutt und Asche legten. (Ondreka 2013, Hervorhebungen von mir)

Und ausgerechnet diese Aussage wird zum Titel des Textes verarbeitet, und zwar in einer vom Kontext losgelösten Form (Sprachforscher Göttert "Das Wort Blockbuster finde ich geschmacklos"), was diesem Gedanken eine Zentralität eingeräumt, die er weder mit Blick auf den Ton noch den Inhalt der Ausführungen haben kann.

In diesen und vergleichbaren Beispielen kommt es also zu einer je nach Medium und Autor mehr oder weniger auffälligen, medienwirksamen Verschmelzung objektivierender linguistischer und subjektivierend-dramatisierender populärwissenschaftlicher Sicht; der versachlichende Beitrag des Linguisten, um auf den Ausdruck Eisenbergs zurückzugreifen, wird also teilweise entsachlicht.

\section{$4 \quad$ Anglizismen in den Spracheinstellungen und im epilinguistischen Diskurs der Sprachen-Studierenden}

\subsection{Untersuchungsziele und Untersuchungsdesign}

Der empirische Teil der Studie betrifft die Wahrnehmung einer Gruppe, die an der Schnittstelle zwischen dem öffentlichen und dem linguistischen Diskurs anzusiedeln ist: Als Sprachbenutzer und linguistische Laien nehmen sie am öffentlichen Diskurs teil, gleichzeitig können sie aufgrund ihrer Studienrichtung als linguistisch vorgebildet bezeichnet werden.

Die Ziele der Erhebung lassen sich in den folgenden Fragen zusammenfassen:

1) Wie ist die Einstellung der Gruppe der sprachinteressierten und linguistisch vorgebildeten Laien, die zugleich angehende/zukünftige Sprachexperten, Lehrer, Übersetzer, Sprachwissenschaftler sind, gegenüber dem gegenwärtigen Zustand und der Vitalität ihrer Sprache?

2) Inwieweit empfinden sie den Gebrauch der Anglizismen in ihrer Sprache als störend und wie verhält sich diese Wahrnehmung zur Einstellung gegenüber Sprachverfall und Sprachvitalität?

3) Wie gestalten sich die epilinguistischen Aussagen und Argumente bezüglich des Anglizismengebrauchs, welche Sprechereigenschaften werden mit ihm assoziiert?

4) Wie verhalten sich die erhobenen Aussagen zum Alter, zur Sprachkompetenz und zur Studienwahl der Studierenden?

5) Inwieweit unterscheiden sich die Einstellungen dieser durch bestimmte biologische und sprachsoziale Parameter (s. u.) definierten Befragtengruppe von den Einstellungen anderer Bevölkerungsgruppen?

Die Erhebung der untersuchungsrelevanten Daten fand im Rahmen eines groß angelegten Forschungsprojekts zu den Spracheinstellungen der italienischen und deutschen Studierenden statt, dessen Ziele und Phasen aus Gründen der Überschaubarkeit im Folgenden kurz skizziert werden:

I. Das Ziel der ersten Befragung (BOLOGNA 2007, vgl. Rocco 2010), die 2007/8 durchgeführt wurde und sich an knapp 500 italienische Studierende richtete, waren die Sprachlernmotivation und die Einstellungen gegenüber dem Deutschen als aktuelle und künftige Verkehrssprache und gegenüber Deutschsprachigen im Vergleich zu anderen Sprachen und Sprechergruppen. 
II. Das zweite, 2013 mit ca. 300 Studierenden durchgeführte Befragungsprojekt fokussierte die Wahrnehmung der aktuellen und zukünftigen Rolle des Deutschen und die Sprachlernmotivation im longitudinalen Vergleich (BOLOGNA 2007 vs. BOLOGNA 2013, vgl. Rocco 2014).

III. Das Ziel des dritten, vom DAAD geförderten und von mehreren Hochschullehrenden ${ }^{14}$ unterstützten Teilprojekts (BOLOGNA 2013 vs. NRW 2013) war es, die Spracheinstellungen der italienischen und deutschen Studierenden zu vergleichen, wobei im Mittelpunkt des Interesses die Frage stand, wie sich die Wahrnehmung der internationalen Verkehrsrolle des Deutschen seitens der italienischen Studierenden zur Perspektive der Muttersprachler verhält. Hierzu wurde der BOLOGNA 2013-Fragebogen ins Deutsche übersetzt und ca. 400 deutschen Studierenden vorgelegt; die Ergebnisse der beiden Befragungen (BOLOGNA 2013, NRW 2013) wurden im Anschluss einander gegenübergestellt.

Die im Folgenden angeführten Items F1-F4, die den Gegenstand der vorliegenden Studie betreffen, nämlich die Wahrnehmung des Zustandes der eigenen Sprache und die Einstellungen zum Anglizismengebrauch, bilden den letzten Teil des o. g. NRW-Fragebogens (Phase III): Die deutsche Fragebogenvariante wurde also um einige Fragen erweitert, die speziell die Einstellungen der Muttersprachler gegenüber den Entwicklungen in ihrer Sprache betreffen:

F1 Die deutsche Sprache droht immer mehr zu verkommen.
A) JA
B) unentschlossen
C) NEIN

F2 Deutsch ist heute lebendiger, vielseitiger als früher.
A) JA
B) unentschlossen
C) NEIN

F3 Der Gebrauch von Anglizismen im Deutschen stört mich.
A) JA
B) je nach Situation
C) NEIN

F4 Der Gebrauch von Anglizismen wirkt meiner Meinung nach eher... (mehrere Antworten/eigene Antwort möglich)
A) kompetent
B) inkompetent
C) freundlich
D) abweisend
E) anziehend
F) abstoßend
G) locker
H) neu
I) jugendsprachlich
J) versnobt
$\mathrm{K})$

F4 ist als eine halboffene Frage konzipiert; neben den zehn angebotenen Antwortkategorien bestand die Möglichkeit, eine eigene Antwort zu formulieren. Die Liste der vorgegebenen Antwortkategorien A bis $\mathrm{J}$ beinhaltet zum einen die in zielverwandten Studien untersuchten bzw. erhobenen epilinguistischen Aussagen (vgl. z. B. Schoel u. a. 2012: 168, 176) und darüber hinaus einige Attribute, denen man im öffentlichen Diskurs über Anglizismen und besonders in Internet-Foren oft begegnet (z. B. "versnobt").

\subsection{Darstellung und Interpretation der Ergebnisse}

Die Befragung wurde zu Beginn der Lehrveranstaltungen an den Universitäten Düsseldorf, Duisburg-Essen, Köln und Bonn durchgeführt. Die Befragtengruppe setzt sich aus 408 Studierenden philologischer Studienrichtungen (v. a. Germanistik/Deutsch, Anglistik und

\footnotetext{
${ }^{14}$ Die Erhebung konnte dank der freundlichen Unterstützung der Professoren Ulrich Ammon, Elmar Schafroth und Dieter Stein durchgeführt werden. Für ihr Entgegenkommen bei der Befragung danke ich auch den Professoren und Dozenten Ulrike Behrens, Albert Bremerich-Vos, Hans Geisler, Karin Ewert-Kling, Martina Nicklaus, Dietmar Osthus, Bernd Rüschoff, Julia Sielaff und Patrick Voßkamp.
} 
Romanistik) zusammen. ${ }^{15}$ Die Studierenden stammen $\mathrm{zu}$ ca. $70 \%$ aus NRW; das Durchschnittsalter beträgt 23 Jahre. ${ }^{16}$ Wie eingangs ausgeführt ist anzunehmen, dass sie als Sprachen-Studierende bereits in Kontakt mit der sprachwissenschaftlichen Sichtweise gekommen waren. Inwiefern sie diese verinnerlicht hatten bzw. verinnerlichen konnten, ist jedoch angesichts der vielen verschiedenen Fächer- und Lehrveranstaltungskombinationen schwer feststellbar; als gemeinsames Merkmal der Gruppe in dieser Hinsicht kann also ein mehr oder minder intensiver Kontakt mit der sprachwissenschaftlichen Perspektive gelten.

Die Häufigkeitsverteilung der Antworten ist in der Tabelle 1 zusammengefasst. Die einzelnen Antwortausprägungen wurden hier aus Übersichtlichkeitsgründen so angeordnet, dass links die positive und rechts die negative Grundhaltung gegenüber dem Zustand des Deutschen und dem Anglizismengebrauch zu finden ist. Die Werte POS und NEG entsprechen der Auswertung und Kategorisierung der Befragtenantworten auf die halboffene Frage F4, die weiter unten beschrieben wird.

\begin{tabular}{|l|l|l|l|}
\hline & $\begin{array}{l}\text { Positive } \\
\text { Grundeinstellung }\end{array}$ & $\begin{array}{l}\text { Neutrale/ } \\
\text { Differenzierende } \\
\text { Grundeinstellung }\end{array}$ & $\begin{array}{l}\text { Negative } \\
\text { Grundeinstellun } \\
\text { g }\end{array}$ \\
\hline $\begin{array}{l}\text { F1 Die deutsche Sprache } \\
\text { droht immer mehr zu } \\
\text { verkommen. }\end{array}$ & NEIN 36\% & unentschlossen 30\% & JA 34 \% \\
\hline $\begin{array}{l}\text { F2 Deutsch ist heute } \\
\text { lebendiger, vielseitiger als } \\
\text { früher. }\end{array}$ & JA 41\% & unentschlossen 35\% & NEIN 24\% \\
\hline $\begin{array}{l}\text { F3 Der Gebrauch von } \\
\text { Anglizismen im Deutschen } \\
\text { stört mich. }\end{array}$ & NEIN 47 \% & je nach Situation 37\% & JA 17\% \\
\hline $\begin{array}{l}\text { F4 Der Gebrauch von } \\
\text { Anglizismen wirkt auf } \\
\text { mich... }\end{array}$ & POS 46\% & MISCH 29\% & NEG 25\% \\
\hline
\end{tabular}

Tab. 1: Zusammenfassung der Antworten bzgl. des Sprachzustandes und des Anglizismengebrauchs.

Erwartungsgemäß besteht zwischen den Antworten auf die beiden Anglizismen betreffenden Fragen eine relativ starke positive Korrelation. ${ }^{17}$ Für die meisten Befragten resultiert, dass ihre Einstellung gegenüber Anglizismen jeweils bei den beiden Fragen positiv (33\%), negativ (12\%) oder neutral (13\%) ausfällt. Den Rest bilden größtenteils die Antwortkombinationen, die aus einer neutralen Ausprägung und einer positiven oder negativen Antwort bestehen (z. B. "je nach Situation" bei F3, POS oder NEG bei F4 oder umgekehrt), was allerdings nicht als inkongruentes Antwortverhalten betrachtet werden kann: Es ist durchaus möglich, dass der Anglizismengebrauch mit eher negativen Attributen beschrieben wird (F4 NEG), jedoch bei der Frage, ob er einen persönlich stört, eine relativierende Antwort gegeben wird (F3 "je nach Situation").

Darüber hinaus wurde untersucht, wie sich die Einstellung gegenüber Anglizismen zur Einstellung gegenüber Sprachzustand (berechnet aufgrund der Antworten auf F1 und F2)

\footnotetext{
15 Die Befragtenzahl 408 entspricht der Anzahl der ausgewerteten Fragebögen unter Ausschluss von ErasmusStudierenden, die sich im Hinblick auf den sprachsozialen Hintergrund wesentlich von der Zielgruppe der Projektphase III - der in Deutschland sozialisierten und lebenden Studierenden - unterscheiden.

16 Das Durchschnittsalter wurde unter Ausschluss von einigen Ausreißerwerten (7 Befragte im Alter zwischen 41 und 73 Jahren) berechnet, die statistisch gesehen den Durchschnittswert verzerren.

17 Der Pearsonsche Korrelationskoeffizient kann hier nur als ein orientativer Wert betrachtet werden (das im Anschluss skizzierte Ergebnis der Kreuzung der beiden Variablen ist im gegebenen Zusammenhang aussagefähiger). Er beträgt 0,6 und deutet somit auf einen statistisch signifikanten positiven Zusammenhang hin.
} 
verhält. Zwischen den beiden Einstellungen besteht ein sehr schwacher positiver Zusammenhang (Pearson 0,2), der jedoch nicht unerwähnt bleiben sollte: Positive Einstellung gegenüber Anglizismen ist tendenziell etwas häufiger bei den Studierenden anzutreffen, die die deutsche Sprache als vital betrachten und die Sprachverfall-These verneinen und umgekehrt. ${ }^{18}$

Der Zusammenhang zwischen dem perzipierten Sprachverfall und der Anglizismenwahrnehmung kann, um auf die Ergebnisse der in Abschnitt 1 erwähnten IDSKonferenz zurückzukommen, in Verbindung mit der Tendenz gesehen werden, "Sprache als etwas 'Homogenes' anzusehen, als etwas, das einer gewissen Stabilität unterliegt, welche sich an Normkodizes (wie zum Beispiel dem Duden) festmachen lässt" (Hein/Mell 2013: 62).

Die Antworten auf die Fragen F1 bis F4 wurden gemäß den in 3.1 skizzierten Zielen auf mögliche Zusammenhänge mit anderen auf die Befragten bezogenen Variablen und Spracheinstellungen überprüft, die im Rahmen des Projekts (vgl. Abschnitt 1, Phase III) erhoben wurden. An dieser Stelle seien nur die wichtigsten Ergebnisse skizziert: Es lässt sich kein statistisch signifikanter Zusammenhang zwischen den untersuchten Einstellungen und Englisch bzw. Deutsch als Studienfach feststellen. Die sehr schwachen Korrelationen zwischen der Variable "Deutsch als Fach" und F3 (Pearson 0,1) sowie "Deutsch als Fach" und F4 $(0,1)$ sind nur insoweit erwähnenswert, da beide dasselbe Vorzeichen haben; $\mathrm{d}$. $\mathrm{h}$. Deutschstudierende zeigen in beiden Fällen eine etwas günstigere Einstellung Anglizismen gegenüber als Studierende, die nicht Deutsch/Germanistik als Fach haben. Es lässt sich ferner eine ebenfalls sehr schwache Korrelation zwischen dem Niveau der Englischkenntnisse und der Wahrnehmung des Anglizismengebrauchs feststellen: Studierende mit sehr guten Englischkenntnissen (aufgrund der Selbsteinschätzung) tendieren etwas häufiger dazu, den Anglizismengebrauch als störend zu bezeichnen $(0,1)$.

Die Antworten auf die halboffene Frage F4 erlauben einen genaueren Einblick in den epilinguistischen Diskurs über Anglizismen. Was das Antwortverhalten betrifft, so haben sogar 76 Befragte (ein Fünftel der Gruppe) die offene Antwortmöglichkeit genutzt, teilweise als Ergänzung zu den bereits angekreuzten Kategorien. Wenn wir die Summe der beiden am häufigsten gewählten Antwortkategorien mit der Gesamtanzahl der Nennungen vergleichen, so resultiert der Anglizismengebrauch als ein Element, das das Sprachverhalten einer Person bzw. Gruppe vor allem diaphasisch und diastratisch markiert: "jugendsprachlich" und "locker" machen insgesamt knapp die Hälfte der gewählten Attribute aus, die die Wirkung des Anglizismengebrauchs beschreiben (384 von insgesamt 877 Nennungen).

Schließt man die Antworten "jugendsprachlich" und "neu", die weder als eindeutig positiv noch als eindeutig negativ betrachtet werden können, aus, so führt die Auszählung zum folgenden Ergebnis: Es wurden insgesamt 303 positiv konnotierte Antworten ("locker" 154 Nennungen, "kompetent" 80, "anziehend" 37, "freundlich" 32) und 145 negativ konnotierte Antworten ("versnobt" 57, "inkompetent" 46, "abstoßend" 25, "abweisend" 17) verzeichnet.

An dieser Stelle können nun die in der Tabelle 1 dargestellten Ausprägungen POS, NEG und MISCH erläutert werden: Die entsprechende Variable wurde unter Berücksichtigung der geschlossenen Antworten A bis J sowie der offenen Antwort K erstellt, die unter folgende Kategorien subsummiert wurden: POS "grundsätzlich positive Bewertung des Anglizismengebrauchs" entspricht den Antwortkombinationen, die sich aus positiv konnotierten und ggf. neutralen Angaben zusammensetzen (z.B. "locker" + "freundlich" oder "locker" + "jugendsprachlich"); NEG "grundsätzlich negative Bewertung" entspricht der Kombination aus negativ konnotierten und ggf. neutralen Antworten. Alle anderen

18 Auch vereinzelt korrelieren die Antworten auf F1und F2 schwach positiv mit den Antworten bezüglich der Anglizismen, die Korrelationskoeffizienten liegen in allen Fällen um 0,2. 
Antwortkombinationen, die sich nicht eindeutig durch die Kategorien positiv/negativ beschreiben lassen, wurden der Kategorie MISCH zugeordnet.

Die Aussagefähigkeit dieser (in der Tabelle 1 veranschaulichten) quantitativen Auswertung soll im Folgenden durch Beispiele ergänzt werden, die die Wortwahl der Studierenden in der Beschreibung der Anglizismenwirkung illustrieren.

Positive Aussagen kreisen um die Konzepte der Weltoffenheit, Internationalität und Flexibilität:

(neu, jug) ${ }^{19}$ tolerant, innovativ

(kompetent, freundlich) weltoffen, interessiert

(anziehend, locker) kommunikativ

(freundlich, locker, jug) bzgl. der Kultur sozial, offen, modern

(kompetent, freundlich) offen für Multikulturalität

(kompetent, locker) flexibel

(locker, jug) aufgeschlossen

(kompetent) offen, flexibel, wissbegierig

(neu) passt sich den Veränderungen international an

(kompetent, anziehend, jugendsprachlich) forschend, international, analysierend

(locker, neu, jug) modern

globalisiert

(locker, jug) internationalisierend

international wichtig

Die o. g. "mittlere" Kategorie (MISCH) bilden einige sachlich-neutrale Antworten, die in Richtung einer linguistischen Argumentation gehen:

linguistisch interessant

ist normaler Ausdruck von Sprachwandel, wie es ihn zu allen Zeiten gegeben hat

(jug) kreativ

(locker, neu, jug) kreativ

neutral, gehören in unserer globalisierten Welt dazu

normal

natürlich

(neu, jug) umgangssprachlicher

(jug) Vereinfachung der Sprache

Zur mittleren Kategorie gehören darüber hinaus relativierende Formulierungen, die tendenziell eine erhöhte Sensibilität für die Kontextabhängigkeit des Sprachgebrauchs zeigen:

(inkompetent) je nach Situation

muss zum Kontext passen

\footnotetext{
${ }^{19}$ Falls die Befragten neben der offenen Antwort auch eine oder mehrere der angebotenen Antwortmöglichkeiten gewählt haben, sind diese in Klammern angegeben; jug steht abkürzend für das oft verzeichnete Attribut "jugendsprachlich". Aus Gründen der Übersichtlichkeit wurden sich wiederholende, unverständliche und schwer kategorisierbare Antworten nicht angeführt.
} 
(jug) je nach Situation kompetent oder inkompetent

abhängig vom Gesprächsthema

kommt auf Sprecher und Gebrauch an

(kompetent, versnobt) Es kommt auf den richtigen Kontext \& Gebrauch an.

im richtigen Kontext: kompetent, sonst übertrieben

(inkompetent) kompetent nur im IT-Bereich

(inkompetent, versnobt) Es sei denn es geht um Marketing - da sehe ich ein, dass vieles nicht eingedeutscht werden kann

(locker, neu, jugendsprachlich) manchmal unangebracht

(jugendsprachlich) im Übermaß negativ

In den eindeutig negativen Kommentaren wird der Anglizismengebrauch mit Profilierungsabsichten, mangelnder (Sprach-)Kompetenz und unkritischer oder unreflektierter Nachahmung fremder Vorbilder assoziiert:

(versnobt) pseudo-modern

(inkompetent, neu, versnobt) künstlich, u. U. angeberisch

(inkompetent, jug) profilierend, nicht wortgewandt

(locker, neu, jug) sinnlos, „einen auf dicke Hose machen“

(locker, jug) teils arrogant

(jug, versnobt) pseudokompetent

(kompetent, abweisend, locker, neu, jug) angeberisch

(locker, jug, versnobt) möchte gern fachsprachlich

(inkompetent, jug) gewollt

(abstoßend, jugendsprachlich) anstrengend

(neu) oft unreflektiert, Bedeutung häufig unklar

(inkompetent, locker, versnobt) zu bequem, um das deutsche Äquivalent zu suchen

unbedarft im Hinblick auf die schöne deutsche Sprache

(abweisend) ungebildet

(locker, jug, versnobt) Anpassung um jeden Preis

Der Anglizismengebrauch wurde ferner mehrfach als unnötig bzw. überflüssig, lächerlich, irritierend und als Gefahr für die deutsche Sprache bezeichnet:

unnötig

(inkompetent, abstoßend, jug) überflüssig

(jug, versnobt) übertrieben/überflüssig

(kompetent) bei Fachsprache/Fachwörtern kompetent, aber oft überflüssig

(inkompetent, abstoßend) lächerlich

(neu) manchmal gewollt und komisch

(jug) verliert an Ernsthaftigkeit

oft lächerlich amerikanisiert

(abstoßend, jug, versnobt) manchmal gut um sich einfacher auszudrücken, oft nervig + unnötig 
(neu, jug) nervig

(neu, jug) irritierend

(abstoßend, jug) nervend, störend \& die deutsche Sprache verstümmelnd

(neu, jug) teilweise unpassend; Angst, dass die deutsche Sprache so verloren geht

(jug) zerstörend für die Sprache

Die Antworten wie "pseudokompetent", "möchte gern fachsprachlich" und "lächerlich" deuten u. a. auf die Wahrnehmung einer Kluft zwischen der Tendenz zur Anglizismenverwendung und den eingeschätzten Englisch- bzw. Fremdsprachenkenntnissen der Bevölkerung hin. Diese Kluft-Empfindung ist sicher auch Teil des öffentlichen Diskurses: In den Medien wird sie in verschiedenen Zusammenhängen thematisiert und oft anhand besonders markiger und $\mathrm{z}$. T. komischer Beispiele karikiert. So weisen z. B. mehrere online frei zugängliche Studien der Endmark AG, die für nationale und internationale Unternehmen Markennamen und Werbeslogans (Claims) entwickelt, darauf hin, dass viele deutsche Unternehmen englische Slogans wählen, die ein Teil des Zielpublikums nicht versteht. Zur Illustration werden eine Reihe misslungener Übersetzungsversuche der Informanten zitiert: z. B. 'Bienen-Inspektion' für Be inspired von Siemens mobile, 'die Fahrt überleben' für Drive Alive von Mitsubishi, 'Wir sind zwei Fahrer' für We are drivers too von Esso (Endmark 2009). In einem Artikel des Karriereportals haufe.de (Haufe Online Redaktion 2011) wird beklagt, dass sich trotz der eher unzureichenden Sprachkenntnisse der Bevölkerung immer mehr Stellenanzeigen mit englischen Berufs- und Stellenbezeichnungen "schmücken". Der Hauptvorwurf lautet, dass Anglizismen in Stellenanzeigen bewusst eingesetzt werden, um wenig attraktive Stellen durch möglichst wichtig klingende englische Vokabeln aufzuwerten.

Die Wortwahl, die die mediale Kritik an der euphemistischen Funktion der Anglizismen kennzeichnet, ist oft ausgeprägt wertend: So prangert ein Artikel aus der Rheinischen Post die Anglizismen ausgehend von einer Stellenanzeige an, bei der für 'Toiletten-Reinigungskraft' WC-Manager benutzt wird. Die betont subjektiv-wertende Haltung ist bereits im Titel ("unsinniges Englisch") sowie an mehreren Textstellen erkennbar: "ein oft lächerlicher englischsprachiger Aufputz von Berufsbeschreibungen", "die Albernheit der sprachlichen Fassadenmalerei", "unechter Sprach-Schmuck", "englisch aufgemotzter Glitzerkram", "modernistisches Imponiergehabe", "Wortprahlerei" (Michels 2009).

Auch die im Datensatz mehrfach auftretende Idee der "Überflüssigkeit" gehört (u. a.) zum öffentlichen Diskurs, der grundsätzlich ungenügend zwischen Sprache und Sprachgebrauch differenziert, wie Eisenberg im zitierten Konferenzbericht betont. Die Überflüssigkeitsthese lässt sich ebenfalls mit linguistischen Argumenten entkräften: Aufgrund des konnotativen Potenzials von Anglizismen, Latinismen usw. und ihrer Funktion in der sozialen Positionierung des Sprechers sowie deren Wahrnehmung kann weder von Identität zwischen dem jeweiligen Anglizismus und dessen deutscher (Teil-)Entsprechung noch von Überflüssigkeit gesprochen werden. Im diesem Zusammenhang ist besonders die Studie von Altleitner (2007) zu erwähnen: Sie veranschaulicht auf breiter empirischer Basis, wie die Bedeutung von Anglizismen oder allgemeiner von Entlehnungen in der Interaktion zwischen Sprecherkognition, Sprachgebrauch, kulturellen und sozialen Faktoren konstruiert wird. ${ }^{20}$

\subsection{Ein vergleichender Blick auf zielverwandte Studien}

Mit Blick auf das Untersuchungsziel 5 (3.1) wurde ein Vergleich vorgenommen mit der 2008 durchgeführten repräsentativen Erhebung (1.820 Befragte), die im Auftrag der Gesellschaft für

\footnotetext{
${ }^{20}$ Durch den Fragebogen der erwähnten Studie werden auch Spracheinstellungen erhoben; es handelt sich jedoch um Sprecherbewertungen in Bezug auf konkrete Beispiele. Die vorliegende Studie konzentriert sich hingegen eher auf allgemeine Einstellungen bzw. Aussagen zum Sprachzustand und zu den Anglizismen.
} 
deutsche Sprache in Zusammenarbeit mit dem Deutschen Sprachrat vom Institut für Demoskopie Allensbach durchgeführt wurde (vgl. Hoberg/Eichhoff-Cyrus/Schulz 2008). ${ }^{21}$ Die Gegenüberstellung der beiden Studien ergab Folgendes:

Die Antworten der Studierenden bezüglich des Anglizismengebrauchs weichen deutlich von den damals ermittelten Werten für ganz Deutschland und für die neuen Bundesländer ab:22 $39 \%$ der befragten Bundesbürger empfinden den Anglizismengebrauch als störend, $40 \%$ als nicht störend, $17 \%$ der Antworten wurden der Kategorie "ganz unterschiedlich" und $4 \%$ der Kategorie "ist mir egal" zugeordnet. Dabei fällt die Akzeptanz für Anglizismen in den alten Bundesländern (37 \% "stört mich", 41 \% "stört mich nicht", 18 \% "ganz unterschiedlich", 4 \% "ist mir egal") etwas höher als in den neuen aus (46 \% "stört mich", 32 \% "stört mich nicht", $17 \%$ "ganz unterschiedlich", 5\% "ist mir egal"). ${ }^{23}$

Beim Vergleich mit den Antworten der jüngeren Bevölkerungsgruppen der GfdS-Studie fällt der Unterschied allerdings bedeutend geringer aus, was für eine entscheidende Rolle des Faktors Alter spricht: Für beide jüngsten Teilgruppen der GfdS-Studie verteilen sich die Antworten wie folgt:

- 16-29 Jahre: "stört mich" 15 \%, "stört mich nicht" 64 \%, "ganz unterschiedlich" 15 \%, "ist mir egal" $6 \%$;

- 30-44 Jahre: "stört mich" 22 \%, "stört mich nicht" 51 \%, "ganz unterschiedlich" 23 \%, "ist mir egal" $4 \%$.

Die Anteile der Befragten, die sich am Anglizismengebrauch stören, sind in beiden Gruppen vergleichbar hoch (17\% in der Studierendengruppe, $15 \%$ in der jüngsten GfdS-Gruppe). Doch es lässt sich feststellen, dass Studierende häufiger relativierende bzw. differenzierende Antwortkategorien gewählt haben ("je nach Situation" in F3: 37 \%, MISCH in F4: 29 \%, "ganz unterschiedlich" in der jüngsten Gruppe der GfdS-Studie: $15 \%$ ), während ein überwiegender Anteil der jüngsten GfdS-Befragten eine entweder positive oder negative Einstellung ausgedrückt hat.

Was das (in beiden Studien gleichlautende) Statement "Die deutsche Sprache droht immer mehr zu verkommen" betrifft, so weichen hier die Studierendenantworten nicht nur vom bundesdeutschen Durchschnitt ab, sondern auch von den Ergebnissen für die beiden jüngsten Gruppen der GfdS-Studie: In der Gesamtgruppe beträgt der Anteil der Personen, die diesem Statement zustimmen, sogar $65 \%$ (gegenüber $23 \%$ der ablehnenden Antworten "sehe das nicht so" und $12 \%$ der Unentschlossenen); eine ähnliche Verteilung wurde auch in der Gruppe der zwischen 30- und 44-Jährigen verzeichnet (63\% zustimmend, $25 \%$ ablehnend, $12 \%$ unentschieden). In der Gruppe zwischen 16 und 29 Jahren ist die Zustimmung zur Sprachverfall-These um $10 \%$ niedriger (53 \% zustimmend, $28 \%$ ablehnend, $19 \%$ unentschieden), jedoch im Vergleich zur Studierendengruppe immer noch recht hoch.

$\mathrm{Zu}$ den ebenfalls zielverwandten Studien von Schoell u. a. (2012), die verschiedene Aspekte der Einstellungen gegenüber Anglizismen gemessen haben, kann aufgrund der großen Unterschiede im Untersuchungsdesign nur ein indirekter und tentativer Vergleich angestellt werden. Das Forscherteam untersuchte u. a., wie die Verwendung von Anglizismen mit den Bewertungsdimensionen Kompetenz und Wärme zusammenhängt. Dabei ermittelten sie, dass

\footnotetext{
21 Diese Umfrage lehnt sich z. T. an eine neun Jahre zuvor veröffentlichte repräsentative Studien, vgl. Stickel/Volz 1999.

22 Die entsprechenden Grafiken können in Schulz (2008) konsultiert werden.

23 Eine noch stärkere Ablehnung wurde in einer von der CASIO Europe GmbH in Auftrag gegebenen, mit 1007 Personen zwischen 14 und 65 Jahren durchgeführten Forsa-Umfrage ermittelt (Forsa 2008): Sogar die Hälfte der Befragten fühlte sich durch den Anglizismengebrauch im Alltag und in der Werbung gestört, wobei auch hier die jüngeren Sprecher der Anglizismenverwendung im Alltag gegenüber positiv eingestellt waren.
} 
sowohl beim Einsatz von direkten als auch beim Einsatz von indirekten Methoden (vgl. Rocco 2010: 29-37) ${ }^{24}$ eine klare Bevorzugung der deutschen Begriffe im Hinblick auf die Kompetenzdimension feststellbar ist, während die Ergebnisse im Hinblick auf die Bewertungsdimension Wärme in Abhängigkeit von der Erhebungsmethode abweichen (bei der direkten, bzw. expliziten Messung, die eher mit dem Erhebungsinstrument der vorliegenden Studie vergleichbar ist, erhielten sowohl deutsche als auch englische Stimuli eine neutrale Bewertung).

Ferner stellten Schoell u. a. (2012) fest, dass die Befragtengruppe bei der expliziten ${ }^{25}$ Kompetenzeinschätzung zwischen verschiedenen Verwendungskontexten unterscheidet: Anglizismen aus dem Berufskontext wurden als relativ kompetent bewertet (die Kompetenzurteile der Nicht-Studierenden fielen dabei allerdings höher aus als die der Studierenden, vgl. ebd.: 179); die Verwendung der Anglizismen im Freizeitkontext wurde im Vergleich zur Verwendung entsprechender deutscher Begriffe als weniger kompetent eingeschätzt. Die Anglizismenverwendung scheint sich also im professionellen Kontext fester etabliert zu haben als im privaten (vgl. ebd.: 177f.) - eine Tendenz, die sich auch in den halboffenen Antworten (F4) der vorliegenden Studie abzeichnet: Nicht nur die zitierten relativierenden Formulierungen, sondern auch die Tatsache, dass sowohl "kompetent" (80 Nennungen) als auch "inkompetent" (46 Nennungen) im Datensatz auftreten, deutet auf eine z. T. kontextabhängige Bewertung des Anglizismengebrauchs hin.

Um noch abschließend auf einige Parallelen mit einer drei Jahrzehnte zuvor durchgeführten Studie hinzuweisen: Die Vorwürfe wie Imponiergehabe, Bequemlichkeit und Unterwürfigkeit, die einige der in 4.2 zitierten Meinungsäußerungen ausdrücken, findet man auch in den Ergebnissen einer der Pionieruntersuchungen auf diesem Gebiet von Stickel (1984). Er untersuchte Leserbriefe und sprachbezogene Glossen aus dem Zeitraum 1979-83 und ermittelte dabei grundlegende Elemente und Argumentationsstrategien des damaligen öffentlichen Anglizismendiskurses. Das aus dem analysierten Material (insbesondere aus den Leserbriefen) resultierende Bild scheint im Vergleich zu den Ergebnissen der GfdS-Studie und besonders der vorliegenden Studie noch bedeutend düsterer zu sein. In diesem Zusammenhang ist aber auch darauf hinzuweisen, dass es sich bei sprachinteressierten Leserbrief-Schreibenden und Autoren von Sprachglossen um Sprechergruppen handelt, die mit der repräsentativen Stichprobe der GfdS und den Befragten dieser Untersuchung nur indirekt vergleichbar sind.

\section{$5 \quad$ Fazit}

Diese Untersuchung hat gezeigt, dass die Spracheinstellungen der Studierenden zwar teilweise von laienlinguistischen Urteilen der öffentlichen Sprachdiskussion geprägt sind. Der Anteil der Studierenden, die zu differenzierten Urteilen tendieren, die Anglizismenverwendung "jenseits von Gut und Böse" betrachten und die These vom Sprachverfall ablehnen, ist jedoch höher als in anderen, im Hinblick auf den Untersuchungsgegenstand vergleichbaren Erhebungen.

Das bedeutet, dass die Zielgruppe, die eine bestimmte, von Einzelfall zu Einzelfall vermutlich je nach Studienlaufbahn variierende sprachwissenschaftliche Vorbildung genossen hat, eine erhöhte Sensibilität für Vielfältigkeit und Dynamik des Sprachgebrauchs hat und einen puristisch oder ideologisch geprägten Fremdwörterdiskurs überwiegend ablehnt.

\footnotetext{
24 Die Autoren sprechen hier von impliziten (nicht vollständig bewussten oder unbewussten) und expliziten Spracheinstellungen bzw. von impliziten und expliziten Maßen. Die Ergebnisse der impliziten Messung können aufgrund der grundlegenden methodischen Unterschiede nicht direkt mit den Ergebnissen der vorliegenden Untersuchung verglichen werden.

25 Vgl. Anmerkung 24.
} 
Wenn wir an dieser Stelle die Ausführungen im ersten Abschnitt einbeziehen, so lässt sich zusammenfassend annehmen, dass sich der dort thematisierte versachlichende Beitrag der Sprachwissenschaft zum öffentlichen Sprachdiskurs zwar je nach sprachpolitischem und gesellschaftlichem Kontext anders gestaltet, ohne institutionelle Unterstützung jedoch eine insgesamt eher geringe Reichweite hat. Das unterstreicht die Rolle, die Schulen und Universitäten spielen (können) in der Herausbildung der Fähigkeit, Phänomene wie Entlehnung, Sprachentwicklung und Sprachvariation sachlich zu beurteilen und in einen breiteren sprachhistorischen Kontext zu stellen. Anders ausgedrückt liegt diese Rolle darin, Sprecher im Allgemeinen und insbesondere auch angehende Lehrer und Sprachexperten zu einer fachkompetenten, von sozialen, politischen und anderen Vorurteilen freien und gleichzeitig ihres Einflusses bewussten Betrachtung sprachlicher Realitäten zu erziehen.

Zum Schluss sei noch im Rückblick auf die Ausführungen in 4.3 die Frage der Erhebungsmethodik angesprochen. Eine sinnvolle Ergänzung der bisherigen Untersuchungen und ein methodisches Desiderat Sinne wäre eine Studie, die zwei Erhebungsansätze kombiniert: einerseits die Erhebung der Bewertungsreaktionen auf allgemeine Statements, die die Sprechereinstellungen und den epilinguistischen Diskurs gegenüber Anglizismen (und ggf. anderen Sprachgebrauchstendenzen) reflektieren, und andererseits die Erhebung der Reaktionen (derselben Informanten) auf Beispiele für jeweiliges Sprachverhalten in konkreten Kontexten (unter Einsatz der Methoden wie Matched-Guise-Technik, Speaker Evaluation Paradigm usw.). Dies setzt zwar ein potenziell eher aufwändiges Erhebungsdesign voraus, das jedoch durch einen hohen Erkenntniszuwachs gerechtfertigt werden kann.

\section{Literatur}

Ágel, Vilmos (2008): "Bastian Sick und die Grammatik. Ein ungleiches Duell". Info DaF 35: 64-84.

Allport, Gordon W. (1935): "Attitudes". In: Murchison, Carl M. (ed.): A handbook of social psychology. Worchester, MA, Clark University Press: 789-844.

Altleitner, Margret (2007): Der Wellness-Effekt. Die Bedeutung von Anglizismen aus der Perspektive der kognitiven Linguistik. Frankfurt a. M.: Peter Lang.

Ammon, Ulrich (2007): "Rückwärtsgewandte Englisch-Gegner". DUZ - Unabhängige deutsche Universitätszeitung 63/5: 9.

Ammon, Ulrich/Eisenberg, Peter/Scholz, Joachim (2008): "Die Rolle der europäischen Sprachen in der Zukunft - Deutsch-russische Erfahrungen und Perspektiven. Drei Konferenzberichte". In: Goethe Institut (ed.): Die Macht der Sprache. Online. München: 66-74. http://www.goethe.de/lhr/pro/mac/Online-Publikation.pdf, letzter Zugriff am 10.07.2014.

Bavoux Claudine (1997): "Constitution et traitement d'un corpus 'cryptoglossique'. Quels fondements théoriques?" In: Frey, Claude/Latin, Danièle (eds.): Le corpus lexicographique. Méthodes de constitution et de gestion. Actes de troisièmes journées scientifiques du réseau thématique de recherche 'Etudes du français en francophonie', A.U.P.E.L.F.-U.R.E.F. Louvain-la-Neuve, De Boeck Université: 71-86.

Braselmann, Petra (2002): "Sprachpflege 'à la française'. Die französische Sprachpolitik im Spiegel der deutschen Presse". Muttersprache 4: 239-308.

Canut, Cécile (1993): "Subjectivité, imaginaires et fantasmes des langues. La mise en discours épilinguistique". Langage et société 93: 71-97.

Canut, Cécile (1998): "Activité épilinguistique et insécurité linguistique". In: Calvet, LouisJean/Moreau, Marie-Louise (eds.): Une ou des normes? Paris, Didier Erudition: 39-48.

Eisenberg, Peter (1999): "Stirbt das Deutsche an den Internationalismen? Zur Integration von Computerwörtern". Der Deutschunterricht 3: 17-24.

Eisenberg, Peter (2011): Das Fremdwort im Deutschen. Berlin: de Gruyter. 
Endmark (ed.) (2009): Endmark-Claim-Studie 2009. https://web.archive.org/web/ 20111016065949/http://www.mc-brandnews.de/fileadmin/Content/Downloads/PDF/ brandnews/Dezember_2009_de/Markenclaims_Endmark_Studie.pdf, letzter Zugriff am 04.08.2014.

Forsa (ed.) (2008): Bekanntheit und Verwendung von Anglizismen. http://www.exword.de/global/downloads/EX-word_Studie_Anglizismen2008.pdf, letzter Zugriff am 31.01.2014.

Gendron, Jean-Denis (1974): "La définition d'une norme de langue parlée au Québec. Une approche sociologique". RLIR 38: 198-209.

Giersberg, Dagmar (2007): "Sprachexperte sieht in Anglizismen keine Gefahr". http://www.welt.de/kultur/article120296426/Sprachexperte-sieht-in-Anglizismen-keineGefahr.html, letzter Zugriff am 31.01.2014.

Gröschel, Bernhard (2009): Das Serbokroatische zwischen Linguistik und Politik. Mit einer Bibliographie zum postjugoslavischen Sprachenstreit. München: Lincom Europea.

Haufe Online Redaktion (2011): "Englische Stellenbezeichnungen verwirren Bewerber". Haufe.de/personal 17.08.2011. http:/www.haufe.de/personal/hr-management/englischestellenbezeichnungen-verwirren-bewerber_80_69946.html, letzter Zugriff am 31.01.2014.

Hein, Katrin/Mell, Ruth M. (2013): "Dynamik und Variation der deutschen Sprache als linguistische Perspektive auf 'Sprachverfall'? Tagungsbericht zur 49. Jahrestagung 'Sprachverfall? Dynamik - Wandel - Variation des Instituts für Deutsche Sprache', 12.14.3.2013, Mannheim". Zeitschrift für Dialektologie und Linguistik 80/1: 60-65.

Hillen, Ingrid S. J. (1982): Untersuchungen zu Kontinuität und Wandel der Sprachpflege im Deutschen Reich, in der Bundesrepublik und in der DDR. Bonn. Dissertation.

Hoberg, Rudolf/Eichhoff-Cyrus, Karin M./Schulz, Rüdiger (2008): Wie denken die Deutschen über ihre Muttersprache und über Fremdsprachen? Wiesbaden: GfdS.

Katz, Daniel/Stotland, Ezra (1959): "A preliminary statement to a theory of attitude structure and change". In: Koch, Sigmund (ed.): Psychology. A study of science. Band 3. New York, McGraw-Hill: 433-475.

Maitz, Péter/Elspaß, Stephan (2007): "Warum der 'Zwiebelfisch' nicht in den Deutschunterricht gehört". Informationen Deutsch als Fremdsprache 34/5: 515-526.

Michels, Reinhold (2006): "Unsinniges Englisch. Gesucht: WC-Manager". RP online 22.10.2009. http://www.rp-online.de/wirtschaft/unternehmen/gesucht-wc-manager-aid1.2324836, letzter Zugriff am 31.01.2014.

Neweklowsky, Gerhard (2010): Die südslawischen Standardsprachen. Wien: Österreichische Akademie der Wissenschaften.

Ondreka, Lukas (2013): "Sprachforscher Göttert: 'Das Wort Blockbuster finde ich geschmacklos"'. Spiegel Online 21.11.2013. http://www.spiegel.de/kultur/gesellschaft/ karl-heinz-goettert-mutter-sprache-deutsch-in-zeiten-der-globalisierung-a-933452.html, letzter Zugriff am 31.01.2014.

Pavić Pintarić, Anita (2003): "Deutsche Lehnwörter im Kroatischen der Lepoglava Region zwischen Purismus und alltäglicher Verwendung". TRANS - Internet-Zeitschrift für Kulturwissenschaften 15/2003. http://www.inst.at/trans/15Nr/06_1/pintaric15.htm, letzter Zugriff am 31.01.2014.

Plümer, Nicole (2000): Anglizismus - Purismus - Sprachliche Identität. Eine Untersuchung zu den Anglizismen in der deutschen und französischen Mediensprache. Frankfurt a. M. etc.: Peter Lang.

Pettersen, Egil (1989): "Die Normierungsarbeit des Norwegischen Sprachrats (norsk språkråd)". In: Fallenstein, Robert/Ropeid, Tor J. (eds.): Sprachpflege in Europäischen Ländern. Bergen, Germanistisches Institut: 35-46. (= Schriften des Germanistischen Instituts der Universität Bergen 11). https://www.sprakradet.no/Toppmeny/Om-oss/English-and-otherlanguages/Deutsch/Die_Normierungsarbeit_des_Nor, letzter Zugriff am 31.01.2014. 
Polenz, Peter von (1967): "Vom Sprachpurismus und Nationalsozialismus. Die 'Fremdwort'Frage gestern und heute". In: Lämmert, Eberhard et al. (eds.): Germanisitik - eine deutsche Wissenschaft. Frankfurt a. M., Suhrkamp: 111-165.

République Française (2014): "Loi n 94-665 du 4 août 1994 relative à l'emploi de la langue française", Art. 3. http://www.legifrance.gouv.fr/affichTexte.do?cidTexte=LEGITEXT 000005616341\&dateTexte=vig, letzter Zugriff am 31.01.2014.

Riegel, Mareike (2007): Sprachberatung im Kontext von Sprachpflege und im Verhältnis zu Nachschlagewerken. Unter besonderer Beachtung der Sprachberatungsstelle des WissenMedia-Verlages. Freiburg i. Br. Dissertation. http://www.freidok.uni-freiburg.de/volltexte /3593/, letzter Zugriff am 31.01.2014.

Rocco, Goranka (2010): Deutsch und Deutschlandbild an einer italienischen Universität. Eine Untersuchung zu den Spracheinstellungen der Studierenden. Rom: Aracne.

Rocco, Goranka (2014): "Sprachlernmotivation, aktuelle und zukünftige Rolle des Deutschen im Vergleich zu anderen Sprachen. Eine Längsschnittstudie zu den Spracheinstellungen der italienischen Studierenden". Deutsche Sprache 02/2014: 168-184.

Samardžija, Marko (1993): Jezični purizam u NDH. Zagreb: Hrvatska sveučilišna naklada.

Sarcher, Walburga Ch. (1994): Über Ideal und Wirklichkeit der Frankophonie. Eine Untersuchung zum Verhältnis und zur sprachlichen Zusammenarbeit zwischen Quebec und Frankreich. Bochum: Brockmeyer.

Schlobinski, Peter (2000): Anglizismen im Internet. (= Network 14). http://www.mediensprache.net/networx/networx-14.pdf, letzter Zugriff am 31.01.2014.

Schneider, Jan Georg (2005): "Was ist ein sprachlicher Fehler? - Anmerkungen zu populärer Sprachkritik am Beispiel der Kolumnensammlung von Bastian Sick". Aptum 2/2005: 154-177.

Schneider, Jan Georg (2006): "Von free-floatendem Kapital, Hardlinern und Instructions. Linguistische Anmerkungen zur populären Anglizismenkritik". Lingua et opinio 2006.

Schneider, Jan Georg (2008): "Das Phänomen Zwiebelfisch - Bastian Sicks Sprachkritik und die Rolle der Linguistik". Der Sprachdienst 4/08: 172-180.

Schoel, Christiane et al. (2012): "Spracheinstellungen aus sozialpsychologischer Perspektive I. Deutsch und Fremdsprachen". In: Eichinger, Ludwig M. et al. (eds.): Sprache und Einstellungen. Spracheinstellungen aus sprachwissenschaftlicher und sozialpsychologischer Perspektive. Mit einer Sprachstandserhebung zum Deutschen von Gerhard Stickel. Tübingen, Narr: 163-203.

Schulz, Rüdiger (2008): "Wie denken die Deutschen über ihre Muttersprache und über Fremdsprachen? Erkenntnisse aus einer repräsentativen Bevölkerungsumfrage 2008". http://www.gfds.de/fileadmin/gfds_download/GfdS_Studie_Spracheinstellung.pdf, letzter Zugriff am 31.01.2014.

Schümann, Michael (2007): "Wer hat Angst vor Bastian Sick? - Das Verhältnis der Sprachwissenschaft zu einem Bestsellerautor und Unterhaltungskünstler". Der Sprachdienst 5/07: 201-208.

Sitta, Horst (1990): "Defizit oder Entwicklung. Zum Sprachstand von Gymnasialabsolventen und Studenten". In: Stickel, Gerhard (ed.): Deutsche Gegenwartssprache. Tendenzen und Perspektiven. Jahrbuch 1989 des Instituts für deutsche Sprache. Berlin/New York, de Gruyter: 233-254.

SpiegelKritik (2006): "Sprachwissenschaftler warnt vor Bastian Sicks 'unverantwortlichen' Deutschtipps". SpiegelKritik. Medienblog für Medienreflexion. http://spiegelkritik.de/2006/ 11/12/sprachwissenschaftler-warnt-vor-bastian-sicks-unverantwortlichen-deutschtipps/, letzter Zugriff am 31.01.2014.

Spitzmüller, Jürgen (2005): Metasprachdiskurse. Einstellungen zu Anglizismen und ihre wissenschaftliche Rezeption. Berlin/New York: de Gruyter. 
Stein, Dieter (2004): Weltsprache Englisch. Dominanz und Beherrschung. Oder: ein bisschen Englisch können viele. Basiert auf einem Vortrag gehalten am 5. Juli 2004 im Industrieklub Düsseldorf in der Reihe "Im Dialog mit der Heinrich-Heine-Universität Düsseldorf". http://www.phil-fak.uni-duesseldorf.de/anglist3/weltsprache_englisch.pdf, letzter Zugriff am 31.01.2014.

Stickel, Gerhard (1984): "Einstellungen zu Anglizismen". In: Besch, Werner et al. (eds.): Festschrift für Siegfried Grosse zum 60. Geburtstag. Göppingen, Kümmerle: 279-310.

Stickel, Gerhard/Volz, Norbert (1999): Meinungen und Einstellungen zur deutschen Sprache. Ergebnisse einer bundesweiten Repräsentativerhebung. Mannheim: Institut für Deutsche Sprache. (= amades -Arbeitspapiere und Materialien zur deutschen Sprache 2/99).

Topalović, Elvira/Elspaß, Stephan (2008): "Die deutsche Sprache ein Irrgarten? Ein linguistischer Wegweiser durch die Zwiebelfisch-Kolumnen". In: Denkler, Markus et al. (eds.): Frischwärts und unkaputtbar. Sprachverfall oder Sprachwandel im Deutschen. Münster, Aschendorff: 37-57.

Upshaw, Harry S. (1975): "Judgement and decision processes in the formation and change of social attitudes". In: Kaplan, Martin F./Schwartz, Steven (eds.): Human judgement and decision processes. New York, Academic Press: 201-228.

Völkl, Sigrid D. (2002): "Bosnisch". In: Krenn, Gerald/Okuka, Milos (eds.): Lexikon der Sprachen des europäischen Ostens. Klagenfurt, Wieser Verlag: 209-218. (= Wieser Enzyklopädie des europäischen Ostens 10).

Wolf, Lothar (1987): Französische Sprache in Kanada. München: Vögel. 